\title{
Anwendungspotential der Impedanzspektroskopie für die Waschlaugensensorik
}

\author{
Uwe Tröltzsch ${ }^{1}$, Roman Gruden ${ }^{2}$, Olfa Kanoun ${ }^{1}$, Andreas Buchholz ${ }^{2}$, Volker Beck ${ }^{2}$
}

Technische Universität Chemnitz ${ }^{1}$

Seuffer $\mathrm{GmbH} \&$ Co. $\mathrm{KG}^{2}$

Professur für Mess- und Sensortechnik

Reichenhainer Straße 70

Bärental 26

09126 Chemnitz

75365 Calw

+4937153137591

+4970516001186

uwe.troeltzsch@etit.tu-chemnitz.de

roman.gruden@seuffer.de

\section{Einleitung}

Die Reinigung von Bekleidung erfolgt im privaten und industriellen Bereich weitgehend vollautomatisch mit Waschvollautomaten. Ziel beim Waschprozess ist es, ein optimales Reinigungsergebnis bei minimalem Einsatz von Wasser, elektrischer und thermischer Energie und Waschmittel zu erzielen. Dafür kommen derzeit hauptsächlich Steuerungsstrategien zum Einsatz, für die durch eine Laborerprobung ein effizienter Ressourceneinsatz ermittelt wurde. Um eine weitere Verbesserung des Waschergebnisses und des Ressourceneinsatzes zu erreichen, können Regelungsalgorithmen zum Einsatz kommen, die jedoch geeignete Messgrößen benötigen. Wichtige Größen sind dabei Wasserhärte, Waschmittelkonzentration und Waschaktivität. Diese Größen sollen online während des Waschprozesses bestimmt werden, um beispielsweise Waschmittel entsprechend der aktuellen Wasserhärte automatisch zu dosieren, Waschmittel bei starker Verschmutzung und abnehmender Waschaktivität automatisch zuzudosieren und den Spülprozess bei vollständig ausgespülten Waschmittelrückständen automatisch zu beenden. Ziel dieses Beitrages ist es, das Potential einer erweiterten Leitfähigkeitsmessung auf der Basis der Impedanzspektroskopie zu zeigen.

\section{Stand der Technik}

Für die Waschlaugensensorik werden allgemein optische und elektrische Verfahren angewendet. Optische Verfahren sind sehr preisgünstig realisierbar. Prinzipiell detektieren optische Verfahren die transmissiven und reflektiven Eigenschaften der Waschlauge [1, 2, 3]. Dabei werden die Trübung und die Veränderung des Brechungsindex als Messeffekt ausgenutzt. Die Messung der Waschmittelkonzentration ist dabei auf höhere Konzentrationen beschränkt, da bei niedrigeren Konzentrationen keine Trübung eintritt. Ebenso erlauben optische Verfahren lediglich die Detektion von Schmutz, der zu einer Trübung führt. Insbesondere organische Verschmutzungen, wie beispielsweise Schweiß, die einen hohen lonenanteil enthalten, können damit nicht detektiert werden. Ebenso erlauben optische Verfahren keine Messung der Wasserhärte. Bei elektrischen, voltametriebasierten Verfahren konnte eine deutliche Konzentrationsabhängigkeit gezeigt werden [4, 5]. Die Datenauswertung ist jedoch aufgrund multivariater Analyseverfahren aufwendig. Die Konzentration ionischer Tenside konnte dabei mit guter Genauigkeit bestimmt werden. Die Untersuchung der Eigenschaften einer Vielzahl verschiedener erhältlicher Waschmittel wurde dabei als wesentliches Kriterium für die Nutzbarkeit des Verfahrens aufgeführt. Die Impedanzspektroskopie als elektrisches Verfahren wurde ebenfalls zur Untersuchung von Tensidlösungen angewendet $[6,7]$. Dabei konnten die Leitfähigkeit und die dielektrischen Eigenschaften mit guter Genauigkeit bestimmt werden [8]. Verfahren auf der Basis der Impedanzspektroskopie nutzen dabei im Wesentlichen optimierte Laboraufbauten. Für die praktische Anwendung ist zusätzlich eine Optimierung des Sensors hinsichtlich Robustheit und Langzeitstabilität erforderlich.

\section{Methodik und Messdatenanalyse}

In diesem Abschnitt wird zunächst die Methodik zur Aufnahme und Auswertung der Messdaten erläutert. Wichtiges Kriterium ist dabei die spätere Implementierung eines Messverfahrens in einem dedizierten 
Messsystem. Dies soll mit einer möglichst einfachen Messschaltung und möglichst ohne aufwendige Signalverarbeitung realisiert werden.

Zur Messung des Impedanzspektrums wird eine Leitfähigkeitsmesszelle (Zellkonstante $0.971 / \mathrm{cm}$ ) direkt mit einem Impedanzanalysator (Agilent 4294A) verbunden und das Impedanzspektrum gemessen. Abbildung 1 zeigt den entsprechenden Messaufbau.

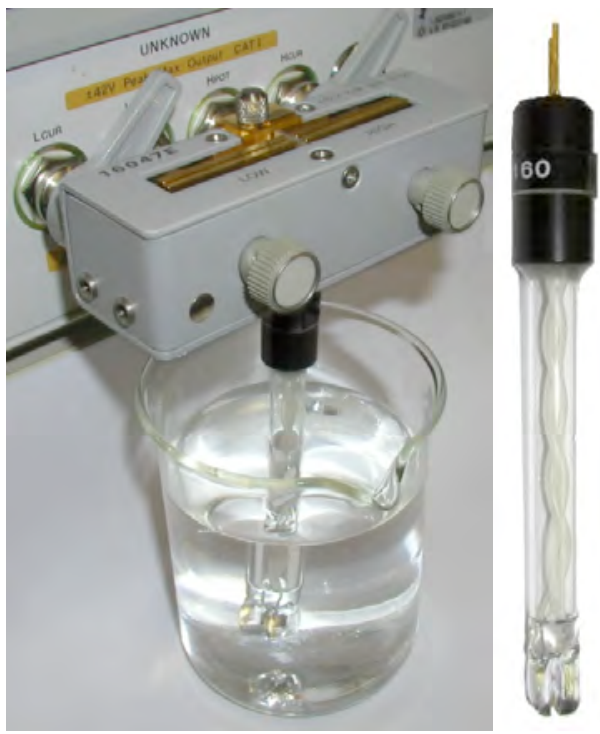

\section{Abbildung 1: Impedanzmessung mit Leitfähigkeitsmesszelle und Agilent 4294A}

Abbildung 2 zeigt ein typisches gemessenes Impedanzspektrum. Dieses zeigt in der Nyqistdarstellung im Bereich $10 \mathrm{kHz}$ bis $100 \mathrm{MHz}$ einen Halbkreis, der bei hohen Frequenzen durch den Koordinatenursprung verläuft. Im Bereich unter $10 \mathrm{KHz}$ ist in der Nyqistdarstellung eine geneigte, nahezu gerade Linie zu beobachten. Zur Charakterisierung des Impedanzspektrums können charakteristische Punkte eingeführt werden, die dessen Eigenschaften weitgehend beschreiben. Der charakteristische Punkt P1 liegt am Maximum des Imaginärteils im unteren Frequenzbereich. Der charakteristische Punkt P2 liegt am Minimum des Imaginärteils im oberen Frequenzbereich. Aus den Impedanz- und Frequenzwerten an den charakteristischen Punkten P1 und P2 lassen sich für eine Waschlauge typische Parameter ableiten.
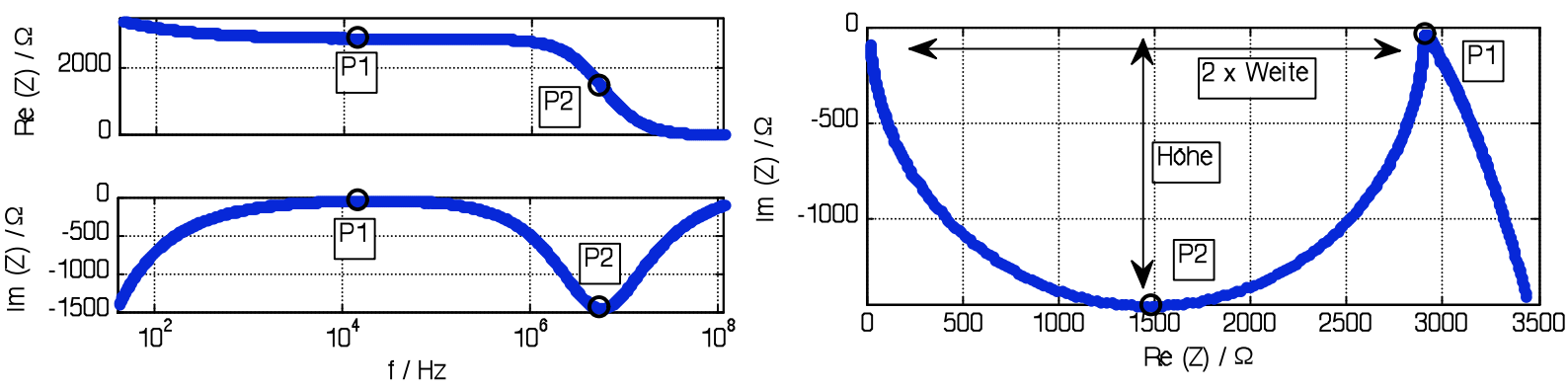

Abbildung 2: Typisches, gemessenes Impedanzspektrum und charakteristische Punkte

Die Leitfähigkeit der Waschlauge lässt sich aus dem Realteil des Punktes $\mathrm{P} 1$ bestimmen, wobei $\mathrm{C}_{\mathrm{z}}$ die Zellkonstante der Leitfähigkeitsmesszelle ist. Durch die Auswertung der Frequenz am Punkt P2 und der Impedanz am Punkt P1 lässt sich die Kapazität der Leitfähigkeitsmesszelle bestimmen. Ein weiteres Maß, das von den Eigenschaften der Waschlauge abhängt, ist die Abflachung des Halbkreises. Die Abflachung kann über das Verhältnis von Höhe zu Weite des Halbkreises bestimmt werden. Mit den folgenden Berechnungsvorschriften lassen sich die entsprechenden Größen bestimmen.

$$
\sigma=\frac{C_{Z}}{\operatorname{Re}\left(Z_{P 1}\right)} \quad C=\frac{1}{2 \pi f_{P 2} \cdot \operatorname{Re}\left(Z_{P 1}\right)} \quad h / w=\frac{-\operatorname{Im}\left(Z_{P 2}\right)}{1 / 2 \cdot \operatorname{Re}\left(Z_{P 1}\right)}
$$

Abbildung 3 zeigt schematisch den Messaufbau und das elekrtrische Ersatzschaltbild. Damit lassen sich die das Impedanzspektrum beschreibenden Merkmale erklären. Die Leitfähigkeitsmesszelle weist zwischen den Messelekroden die Kapazität $C_{1}$ und zwischen den Zuleitungen die Kapazität $C_{2}$ auf. Weiterhin wirkt zwischen den Messelektroden die Leitfähigkeit des Elektrolyten in Form eines Widerstandes $\mathrm{R}_{\mathrm{E}}$. Da in den metallischen Zuleitungen Ladungen als Elektronen und im Elektrolyten 
Ladungen als Ionen transportiert werden muss an der Elektrodenoberfläche ein Ladungsaustausch in Form einer Durchtrittsreaktion stattfinden, der auch mit Diffusionsprozessen verbunden sein kann. Diese Impedanz, die eine Elektrodenpolarisation hervorruft, kann durch die komplexen Impedanzen $Z_{1}$ und $Z_{2}$ beschrieben werden. Relevant für die Entstehung des Halbkreises im Impedanzspektrum ist die Interaktion der Kapazität der Messelektroden und dem Elektrolytwiderstand. Relevant für die geneigte, nahezu gerade Linie ist die Elektrodenpolarisation. Ein ähnliches Modell wurde bereits zur Charakterisierung von Elektroden genutzt [9].

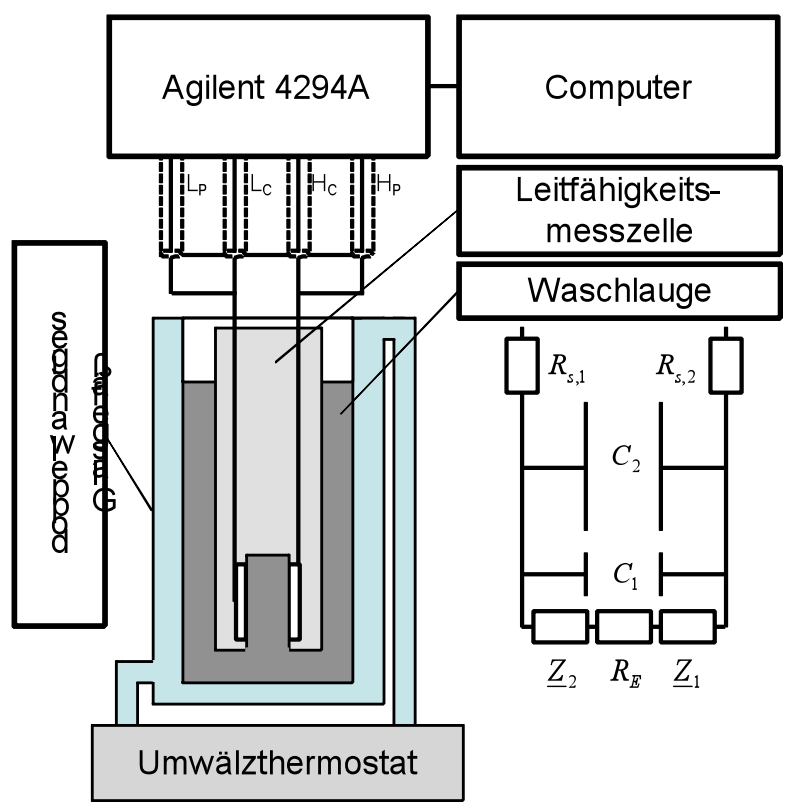

\section{Abbildung 3: Schematische Darstellung von Messaufbau und Impedanzmodell}

Anhand einer Messung an einer KCl-Lösung bei verschiedenen Konzentrationen kann die Plausibilität des Impedanzmodells gezeigt werden. Wird die Leitfähigkeit der Lösung aus dem Realteil des Punktes P1 und der Zellkonstante bestimmt, ergibt sich der in Abbildung 4 gezeigte Verlauf.
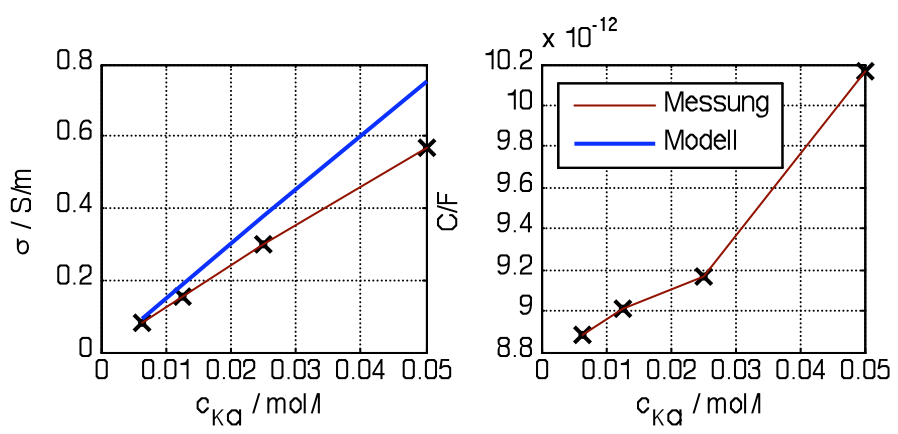

\section{Abbildung 4: Prüfung von Leitfähigkeit und Zellkapazität mit KCl-Lösung}

Mit Hilfe des Kohlrauschgesetzes kann der für die Leitfähigkeit zu erwartende Wert aus der Äquivalentleitfähigkeit bei $25^{\circ} \mathrm{C}$ berechnet werden [10].

$$
\sigma_{\text {Modell }}=c\left(\lambda_{+}+\lambda_{-}\right) \text {mit } \lambda_{+}=\lambda_{K^{+}}=73,5 \frac{\mathrm{S} \mathrm{cm}^{2}}{m} \text { und } \lambda_{-}=\lambda_{C^{-}}=76,4 \frac{\mathrm{S} \mathrm{cm}^{2}}{m}
$$

In Abbildung 4 ist die nach dem Kohlrauschgesetz berechnete Leitfähigkeit $\sigma_{\text {Modell }}$ ebenfalls dargestellt. Dabei zeigt sich eine Abweichung von etwa 20\%. Die Ursachen für die Abweichung liegen hauptsächlich in der Methode zur Präparation der KCl Lösung, der Reinheit des genutzten Salzes und der Genauigkeit der verwendeten Temperaturmessung. Zur Herstellung der Lösung wurde $\mathrm{KCl}$ abgewogen und in destilliertem Wasser gelöst. Zum Einstellen geringerer Konzentrationen wurde die Lösung durch Zugabe von destilliertem Wasser verdünnt. Dabei treten Abweichungen beim Abwiegen des destilliertem Wassers und des $\mathrm{KCl}$-Salzes von etwa je 5\% auf. Eine weitere Rolle spielt der Temperatureinfluss. Die Temperatur der Lösung wurde mit einer Genauigkeit von 2K bestimmt. Bei einem Temperaturkoeffizienten von etwa $2 \% / \mathrm{K}$ ergibt sich eine weitere relative Abweichung von etwa $4 \%$. Da 
das genutzte destillierte Wasser und das genutzte $\mathrm{KCl}$ Salz keiner besonderen Behandlung zur Verbesserung der Reinheit unterzogen wurden ist eine Abweichung der Leitfähigkeit von $20 \%$ plausibel.

Zur Verifikation des Kapazitätswertes wurde an der Leitfähigkeismesszelle eine Kapazitätsmessung ohne Lösung in Luft durchgeführt. Die dabei gemessene Kapazität entspricht dem Wert der Parallelschaltung von $\mathrm{C}_{1}$ und $\mathrm{C}_{2}$. Mit Lösung ergibt sich nach Abbildung 4 eine mittlere Kapazität von 9,5pF. In diesem Fall entspricht die relative Dielektrizitätskonstante der Kapazität $\mathrm{C}_{1}$ der relativen Dielektrizitätskonstante von Wasser. Damit kann das folgende Gleichungssystem mit den beiden unbekannten $\mathrm{C}_{1}$ und $\mathrm{C}_{2}$ aufgestellt werden.

$$
\begin{aligned}
& \text { Luf t. } C=2,125 p F=C_{1}+C_{2} \\
& K C l: C=9,5 p F=\varepsilon_{r} C_{1}+C_{2} \quad \text { mit } \varepsilon_{r}=80
\end{aligned}
$$

Die Lösung des Gleichungssystems ergibt die folgenden Werte.

$$
C_{1}=0,0934 \mathrm{pF}, C_{2}=2,0316 \mathrm{pF}
$$

Bei bekannter Zellkonstante $C_{z}$ kann der Wert der Kapazität $C_{1}$ berechnet werden.

$$
C_{1, \text { Modell }}=\varepsilon_{r} \frac{\varepsilon_{0}}{C_{Z}}=0,091279 p F \quad \text { mit } \varepsilon_{r}=1 \text { und } C_{Z}=0.97 \frac{1}{\mathrm{~cm}}
$$

Der Vergleich des aus den Messdaten bestimmten Kapazitätswertes für $\mathrm{C}_{1}$ mit dem nach dem Modell eines Plattenkondensators bestimmten Wertes zeigt eine sehr gute Übereinstimmung bei einer Abweichung von etwa 2\%. Insgesamt kann damit die Gültigkeit des Impedanzmodells gezeigt werden.

\section{Ergebnisse und Diskussion}

Das Impedanzspektrum von Wasser verschiedenen Ursprungs und von Waschlaugen mit verschiedenen Waschmittelkonzentrationen bei verschiedenen Temperaturen wurde untersucht. Ziel ist dabei die Identifikation der Abhängigkeiten von Wasserhärte und Waschmittelkonzentration, um daraus Messverfahren zu deren Messung ableiten zu können. Sofern Wasserhärte und Waschmittelkonzentration gemessen werden können besteht damit die Möglichkeit den Waschprozess zu Optimieren indem beispielsweise Waschmittel automatisch dosiert und der Spülprozess geregelt werden kann.

\subsection{Einfluss der Wasserhärte}

Die Leitfähigkeit des Wassers zeigt eine sehr ausgeprägte und systematische Abhängigkeit von Wasserhärte und Temperatur. Wie in Abbildung 5 gezeigt, steigt mit zunehmender Wasserhärte, was einer steigenden lonenkonzentration entspricht, auch die Leitfähigkeit. Ebenso führt, wie in Abbildung 6 gezeigt, ein Temperaturanstieg zu einer erhöhten Leitfähigkeit durch die erhöhte lonenbeweglichkeit. Die Kapazität und das Verhältnis von Höhe zu Weite zeigen dabei keine systematische Abhängigkeit von der Wasserhärte. Zu beachten ist, dass das Verhältnis von Höhe zu Weite nahezu 1 ist. Das heißt, dass im Impedanzspektrum ein nahezu idealer Halbkreis zu beobachten ist.
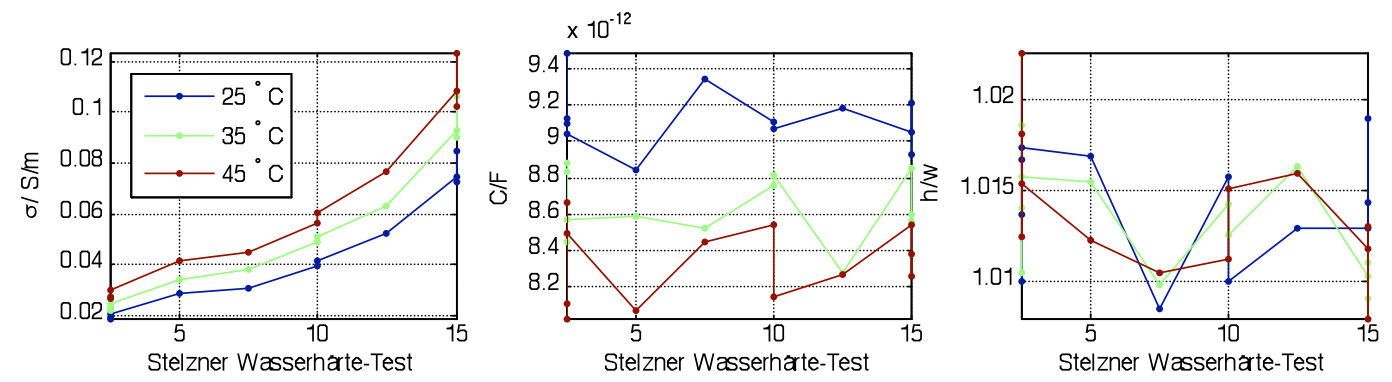

Abbildung 5: Abhängigkeit von der Wasserhärte 

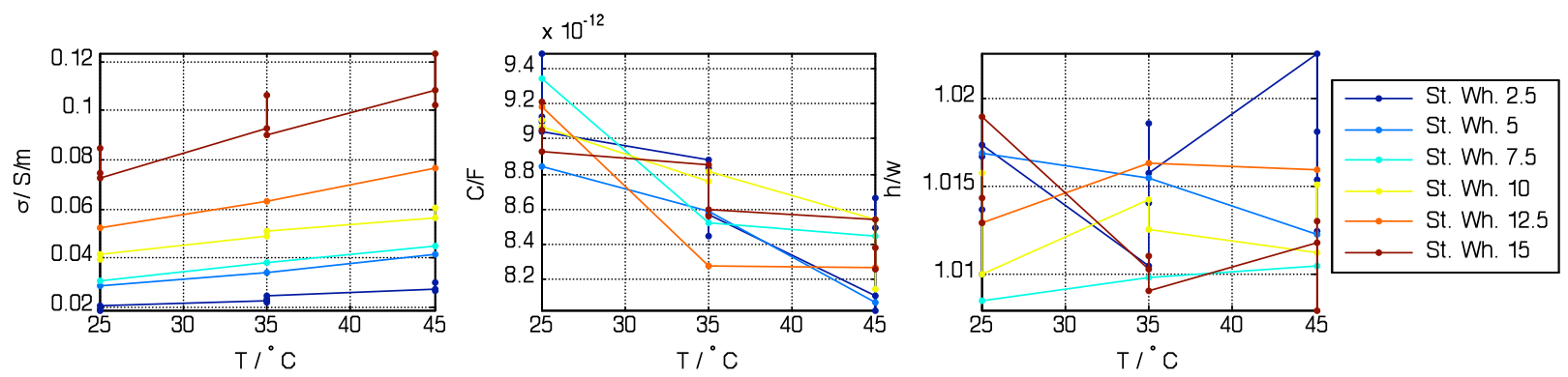

Abbildung 6: Temperaturabhängigkeit

Aufgrund der beobachteten Abhängigkeit der Leitfähigkeit kann für diese ein lineares Modell für die Temperaturabhängigkeit und die Abhängigkeit vom Wert $S$ des Stelzner Wasserhärte-Tests angenommen werden.

$$
\sigma=\Lambda^{\prime} S\left(1+\alpha_{25, \Lambda^{\prime}}\left(T-T_{0}\right)\right)+\sigma_{0}\left(1+\alpha_{25, \sigma_{0}}\left(T-T_{0}\right)\right) \quad m i t T_{0}=25^{\circ} C
$$

Der Wert $\sigma_{0}$ beschreibt dabei die Leitfähigkeit bei einem Wert von $\mathrm{S}=0, \alpha_{25, \sigma_{0}}$ ist der dazugehörige Temperaturkoeffizient des Nullpunktes. Der Wert $\Lambda^{\prime}$ ist vergleichbar zur lonenäquivalentleitfähigkeit jedoch nicht bezogen auf eine bestimmte Konzentration sondern direkt auf den Messwert des Stelzner Wasserhärte-Tests. Der Wert $\alpha_{25, \Lambda^{\prime}}$ beschreibt den dazugehörigen Temperaturkoeffizienten. Für eine Messreihe mit $n$ Messwerten kann ein lineares Gleichungssystem aufgestellt werden, durch dessen Lösung sich die Parameter dieses linearen Modells berechnen lassen.

$$
\left(\begin{array}{c}
\sigma_{1} \\
\mathrm{M} \\
\sigma_{n}
\end{array}\right)=\left(\begin{array}{cccc}
S_{1} & S_{1}\left(T_{1}-T_{0}\right) & 1 & \left(T_{1}-T_{0}\right) \\
\mathrm{M} & \mathrm{M} & \mathrm{M} & \mathrm{M} \\
S_{n} & S_{n}\left(T_{n}-T_{0}\right) & 1 & \left(T_{n}-T_{0}\right)
\end{array}\right) \cdot\left(\begin{array}{c}
\Lambda^{\prime} \\
\Lambda^{\prime} \alpha_{25, \Lambda^{\prime}} \\
\sigma_{0} \\
\sigma_{0} \alpha_{25, \sigma_{0}}
\end{array}\right)
$$

Bei Lösung des Gleichungssystems ergeben sich die folgenden Parameter. Insbesondere die Temperaturkoeffizienten stimmen dabei sehr gut mit dem erwarteten Wert überein. Von verdünnten Salzlösungen und natürlichen Wässern beträgt dieser etwa $\% / \mathrm{K}$.

$$
\Lambda^{\prime}=0.004406 \frac{\mathrm{S}}{\mathrm{m}}, \alpha_{25, \Lambda^{\prime}}=2,19 \frac{\%}{\mathrm{~K}}, \sigma_{0}=0.005356 \frac{\mathrm{S}}{\mathrm{m}}, \alpha_{25, \sigma_{0}}=2,225 \frac{\%}{\mathrm{~K}}
$$

Mit den berechneten Parametern kann ein Vergleich zwischen gemessener und anhand des Modells berechneter Leitfähigkeit durchgeführt werden. In Abbildung 7 ist dabei eine sehr gute Übereinstimmung der Systematik zu beobachten.

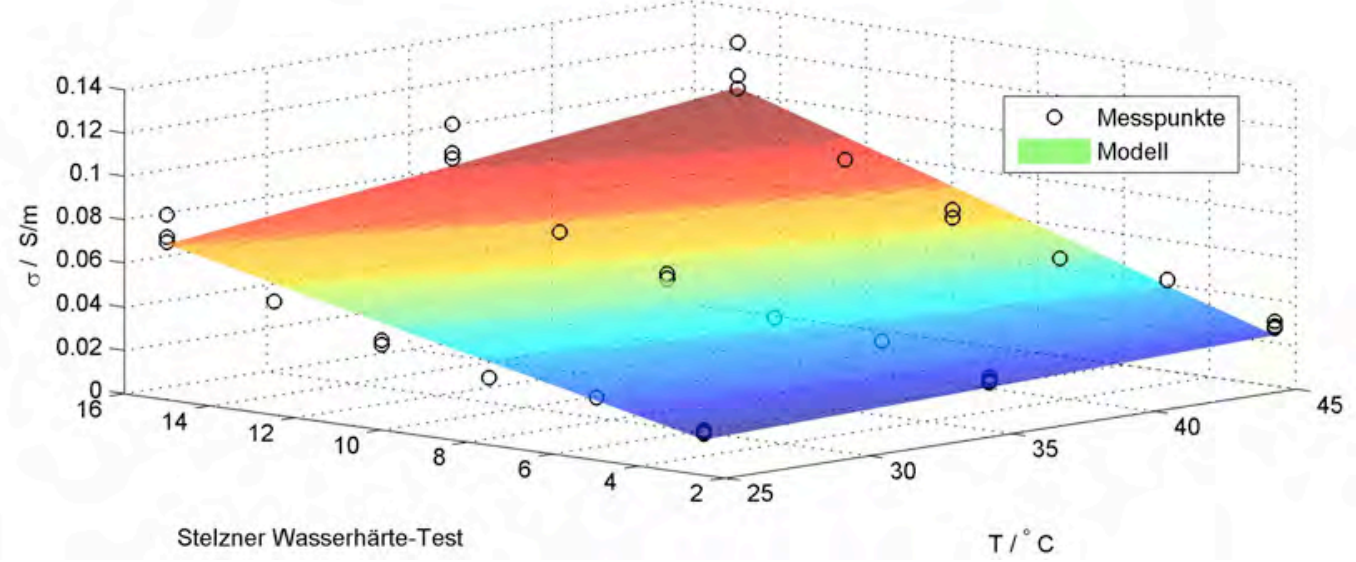

Abbildung 7: Vergleich zwischen gemessenen und berechneten Leitfähigkeiten

Bei bekannten Parametern kann nun aus einem Messwert für die Leitfähigkeit der Wert $S_{\text {berechnent }}$ des Stelzner Wasserhärte-Tests berechnet werden. Die Qualität des Ergebnisses lässt sich anhand der relativen Abweichung zwischen dem berechneten Wert und dem wahren Wert beurteilen. 


$$
\begin{gathered}
S_{\text {berechnet }}=\frac{\sigma-\sigma_{0}\left(1+\alpha_{25, \sigma_{0}}\left(T-T_{0}\right)\right)}{\Lambda^{\prime}\left(1+\alpha_{25, \Lambda^{\prime}}\left(T-T_{0}\right)\right)} \\
f_{\text {rel }}=\frac{S_{\text {berechnet }}-S}{S}
\end{gathered}
$$

In Abbildung 8 ist die relative Abweichung bei der Wasserhärtemessung aus der Leitfähigkeit gezeigt. Insbesondere mittlere Wasserhärten können dabei mit einer guten Genauigkeit von unter $20 \%$ bestimmt werden. Da der Stelzner Wasserhärte-Test selbst mit einer absoluten Abweichung von etwa $\pm 1,25$ behaftet ist, was bei einem Wert von 2,5 einer relativen Abweichung von 50\% entspricht, kann geschlussfolgert werden, dass die gezeigte Methodik sehr gut zur Wasserhärtebestimmung für die Waschlaugensensorik geeignet ist. Der Temperatureinfluss kann nahezu perfekt kompensiert werden. Die relative Abweichung zeigt keine ausgeprägte Temperaturabhängigkeit. Aufgrund der guten Ergebnisse müssen die Werte für Kapazität und dem Verhältnis von Höhe zu Weite nicht für die Wasserhärtebestimmung herangezogen werden.

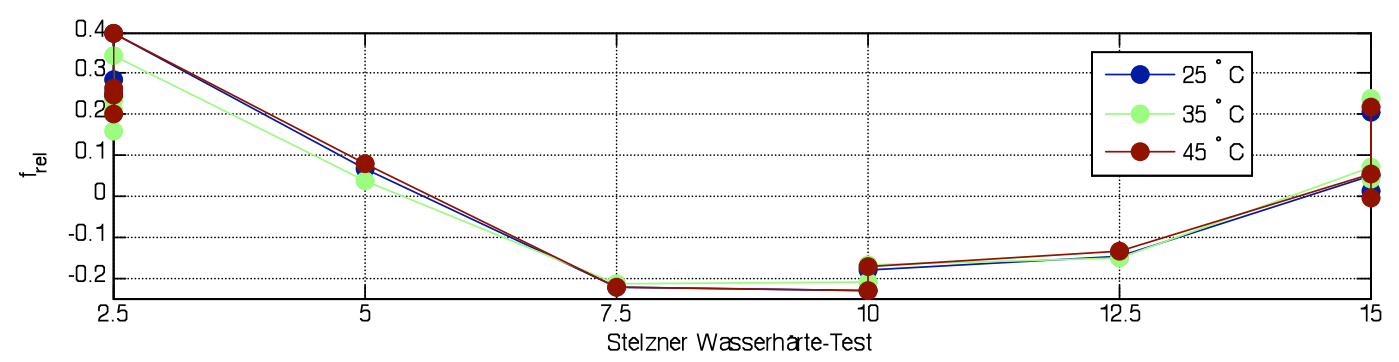

Abbildung 8: relative Abweichung bei der Wasserhärtebestimmung aus der Leitfähigkeit

\subsection{Einfluss der Waschmittelkonzentration}

Ein wesentlicher Faktor für die Waschlaugensensorik ist die Bestimmung der Waschmittelkonzentration. Dies ist beispielsweise für die Regelung des Spülprozesses erforderlich. Der Spülprozess muss nur so lange fortgesetzt werden, bis eine bestimmte Waschmittelkonzentration unterschritten ist. Dabei spielt der Temperatureinfluss eine wichtige Rolle. Ebenso können beim wiederholten Erhitzen und Abkühlen einer Waschlauge Hystereseeffekte auftreten. Deren Einfluss soll ebenfalls quantitativ untersucht werden.

\subsubsection{Einfluss der Temperatur bei wiederholtem Erhitzen und Abkühlen}

Um den Einfluss des wiederholten Erhitzens und Abkühlens zu untersuchen wurden Untersuchungen an reinem Wasser und einer Waschlauge bei der empfohlenen Waschmittelkonzentration durchgeführt. Die Flüssigkeit wurde von Raumtemperatur auf $90^{\circ} \mathrm{C}$ erhitzt und danach wieder auf Raumtemperatur abgekühlt. Der Temperaturzyklus wurde insgesamt dreimal durchlaufen. Abbildung 9 zeigt die Messergebnisse für reines Wasser und Abbildung 10 die Ergebnisse für die Waschlauge. Dabei sind farblich unterschieden die Ergebnisse in Vorwärtsrichtung und Rückwärtsrichtung gezeigt. Wie erwartet nimmt die Leitfähigkeit mit steigender Temperatur zu. Die Kapazität zeigt keine eindeutige Tendenz. Das Verhältnis von Höhe zu Weite sinkt nur mit Waschmittel sehr stark mit zunehmender Temperatur.
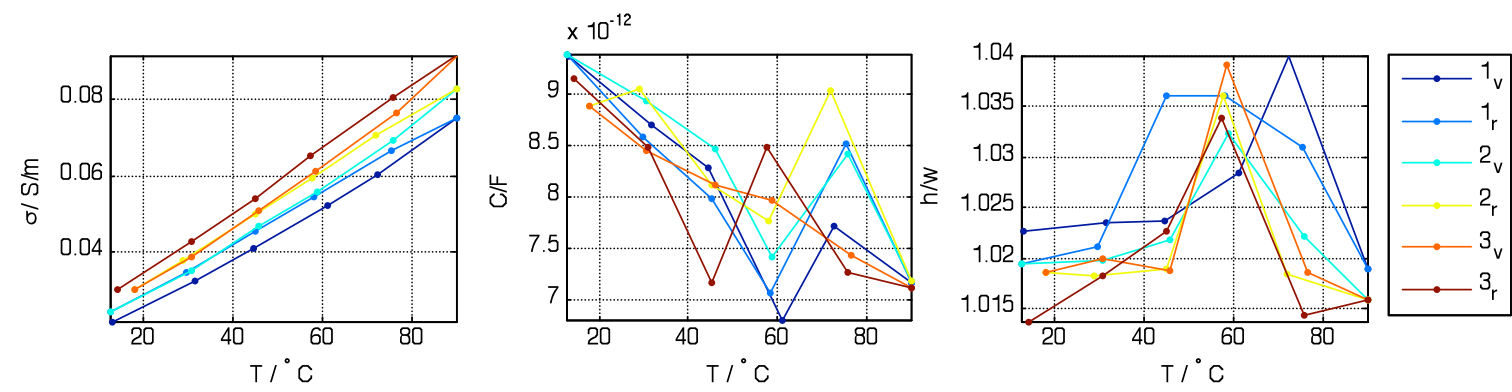

Abbildung 9: Hysterese bei wiederholtem Erhitzen und Abkühlen ohne Waschmittel 

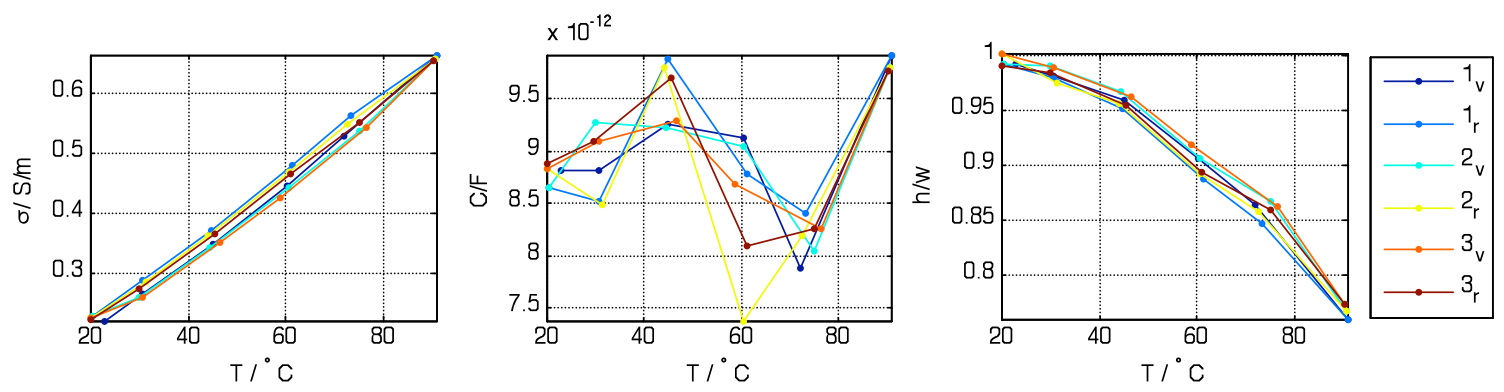

\section{Abbildung 10: Hysterese bei wiederholtem Erhitzen und Abkühlen mit $0.00406 \mathrm{~g} / \mathrm{g}$ Waschmittel}

Für die Leitfähigkeit kann ein temperaturabhängiges, lineares Modell nach folgender Gleichung angenommen werden.

$$
\sigma=\sigma_{0}\left(1+\alpha_{25, \sigma_{0}}\left(T-T_{0}\right)\right) \text { mit }_{0}=25^{\circ} \mathrm{C}
$$

Anhand der Messdaten lassen sich durch die Lösung eines linearen Optimierungsproblems vergleichbar zum vorangegangenen Abschnitt die Parameter des linearen Modells bestimmen. Abbildung 11 zeigt die Ergebnisse. Die Temperaturkoeffizienten liegen dabei zwischen 2,1\%/K und 2,9\%/K. Das liegt im für verdünnte Salzlösungen typischen Bereich. Eine klare Tendenz ist in den ersten drei Zyklen nicht zu beobachten. Der Temperaturkoeffizient scheint mit zunehmender Zyklenzahl tendenziell zu steigen. Die Werte in Rückwärtsrichtung sind niedriger als die Werte in Vorwärtsrichtung. Der Nullpunkt $\sigma_{0}$ steigt für das reine Wasser und fällt tendenziell für die Wachlauge. Die Werte in Rückwärtsrichtung sind höher als die Werte in Vorwärtsrichtung.
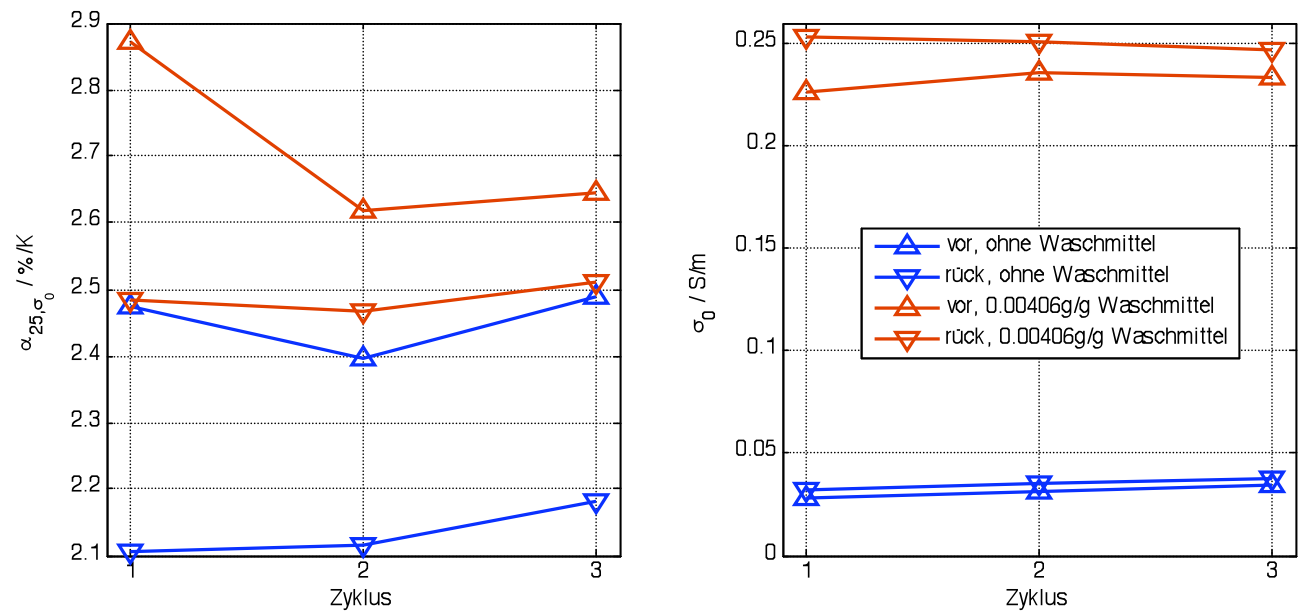

Abbildung 11: Analyse der Leitfähigkeit der Hysterese

Eine statistisch belegbare Aussage kann anhand der vorliegenden Daten nicht getroffen werden. Es kann vermutet werden, dass sich nach einer gewissen Zyklenzahl stabile Endwerte einstellen. Dazu ist in zukünftigen Untersuchungen das Durchfahren einer größeren Zyklenzahl notwendig.

\subsubsection{Einfluss der Waschmittelkonzentration}

Der Einfluss der Waschmittelkonzentration wurde bei $45^{\circ} \mathrm{C}$ und $90^{\circ} \mathrm{C}$ genauer untersucht. Die Leitfähigkeit steigt dabei linear mit der Konzentration wie nach dem Kohlrauschgesetz erwartet und weist einen positiven Temperaturkoeffizienten auf. Die Kapazität der Leitfähigkeitsmesszelle steigt mit der Ionenkonzentration. Ein ähnliches Verhalten wurde ebenfalls in [7] beobachtet und auf veränderte Polarisationseigenschaften der Wassermoleküle zurückgeführt. Die Messwerte für die Kapazität weisen eine strake Streuung auf. Das Verhältnis von Höhe zu Weite sinkt sehr stark mit zunehmender Waschmittelkonzentration. Mit zunehmender Temperatur nimmt der Effekt zu (Abbildung 10 und Abbildung 12). Zurückzuführen ist dies auf eine zunehmende Inhomogenität der Waschlauge da mit zunehmender Temperatur und zunehmender Waschmittelkonzentration mehr verschiedene lonen mit unterschiedlichen Löslichkeiten in die Lösung gelangen. Die Zeitkonstante aus Leitfähigkeit und dielektrischen Eigenschaften ist in diesem Fall nicht mehr diskret sondern verteilt durch die Überlagerung mehrerer Zeitkonstanten unterschiedlicher Ionen. Insbesondere dieser Effekt kann über die Leitfähigkeitsmessung hinaus für die Messung der Waschmittelkonzentration genutzt werden. 

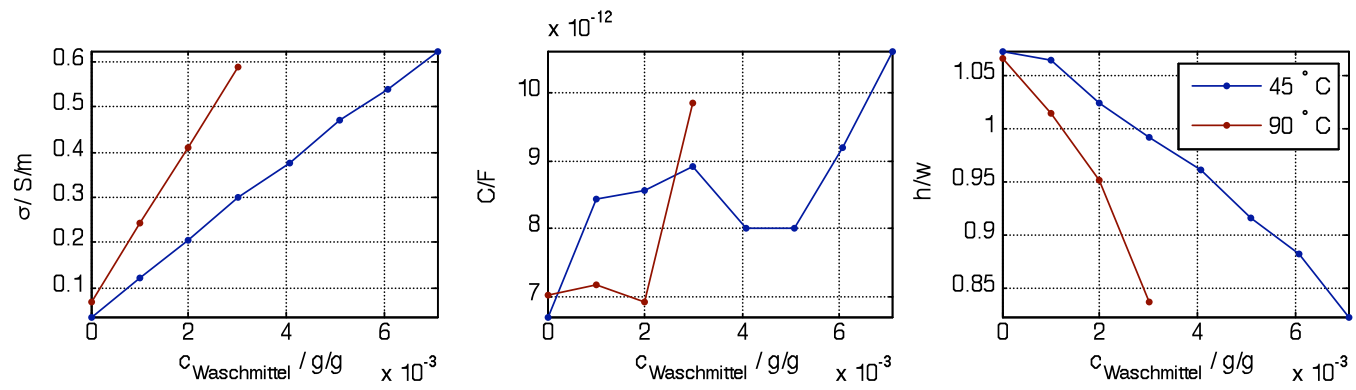

Abbildung 12: Abhängigkeit von der Waschmittelkonzentration

Für die Leitfähigkeit kann erneut das bereits für die Wasserhärtebestimmung genutzte in Waschmittelkonzentration und Temperatur lineare Modell genutzt werden, um die Konsistenz der Daten zu prüfen.

$$
\sigma=\Lambda c_{\text {Waschmittel }}\left(1+\alpha_{25, \Lambda}\left(T-T_{0}\right)\right)+\sigma_{0}\left(1+\alpha_{25, \sigma_{0}}\left(T-T_{0}\right)\right) \text { mit } T_{0}=25^{\circ} \mathrm{C}
$$

Aus den Messdaten für die Leitfähigkeit in Abbildung 12 können die folgenden Parameter bestimmt werden.

$$
\Lambda=43,2470 \frac{\mathrm{S}}{\mathrm{m}} / \frac{\mathrm{g}}{\mathrm{g}}, \alpha_{25, \Lambda}=4,64 \frac{\%}{\mathrm{~K}}, \sigma_{0}=0,0296 \frac{\mathrm{S}}{\mathrm{m}}, \alpha_{25, \sigma_{0}}=1,94 \frac{\%}{\mathrm{~K}}
$$

Der Temperaturkoeffizient für die Ionenäquivalentleitfähigkeit ist dabei sehr groß im Vergleich zum erwarteten Wert von etwa $2 \% / \mathrm{K}$. Da nur zwei Temperaturpunkte vorliegen ist diese Abweichung akzeptabel. Die Leitfähigkeit am Nullpunkt stimmt sehr gut mit dem erwarteten Wert von etwa 0,03 S/m überein. Diese Leitfähigkeit entspricht einer berechneten Wasserhärte von 6,7 und stimmt damit gut mit dem gemessenen Wert von 5 überein.

\subsubsection{Kombinierter Temperatur und Waschmitteleinfluss}

Durch die Kombination der Daten aus den vorangegangenen Abschnitten kann mit Hilfe der Leitfähigkeit ein erster Ansatz zur Ermittlung der Waschmittelkonzentration gezeigt werden. Abbildung 13 zeigt die Leitfähigkeit in Abhängigkeit von Temperatur und Waschmittelkonzentration. Dabei sind die Daten für die Leitfähigkeit aus Abbildung 9, Abbildung 10 und Abbildung 12 zusammengefasst.
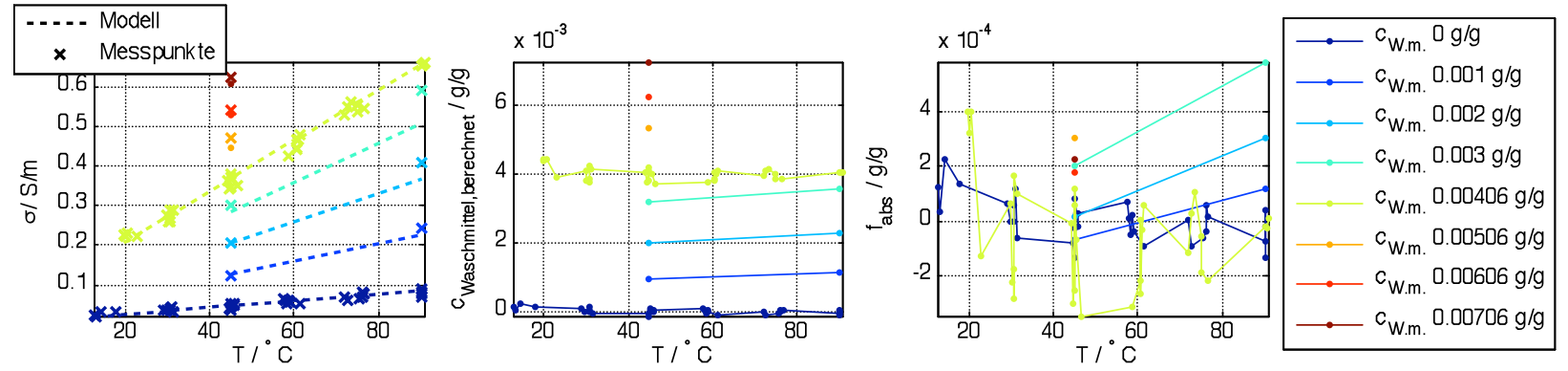

Abbildung 13: Berechnung der Waschmittelkonzentration

Unter Nutzung aller Messpunkte lässt sich das Leitfähigkeitsmodell erneut parametrieren. Dabei ergeben sich die folgenden Parameter. Die Temperaturkoeffizienten entsprechen dabei sehr gut den typischen Werten von etwa $2 \% / \mathrm{K}$. Die Leitfähigkeit am Nullpunkt entspricht exakt dem erwarteten Wert, der einer berechneten Wasserhärte von 6,9 entspricht.

$$
\Lambda=51,77 \frac{\mathrm{S}}{\mathrm{m}} / \frac{\mathrm{g}}{\mathrm{g}}, \alpha_{25, \Lambda}=2,65 \frac{\%}{\mathrm{~K}}, \sigma_{0}=0,0304 \frac{\mathrm{S}}{\mathrm{m}}, \alpha_{25, \sigma_{0}}=2,81 \frac{\%}{\mathrm{~K}}
$$

Durch Auflösen des Leitfähigkeitsmodells nach der Waschmittelkonzentration kann diese bei bekannter Temperatur aus dem Messwert für die Leitfähigkeit berechnet werden.

$$
c_{\text {Waschmittel,berechnet }}=\frac{\sigma-\sigma_{0}\left(1+\alpha_{25, \sigma_{0}}\left(T-T_{0}\right)\right)}{\Lambda\left(1+\alpha_{25, \Lambda}\left(T-T_{0}\right)\right)}
$$


Die in Abhängigkeit von der Temperatur berechneten Werte für die Waschmittelkonzentration und die absolute Abweichung vom tatsächlichen Wert sind ebenfalls in Abbildung 13 dargestellt. Die maximale Abweichung liegt bei etwa $410^{-4} \mathrm{~g} / \mathrm{g}$, was etwa $10 \%$ der empfohlenen Waschmittelkonzentration entspricht.
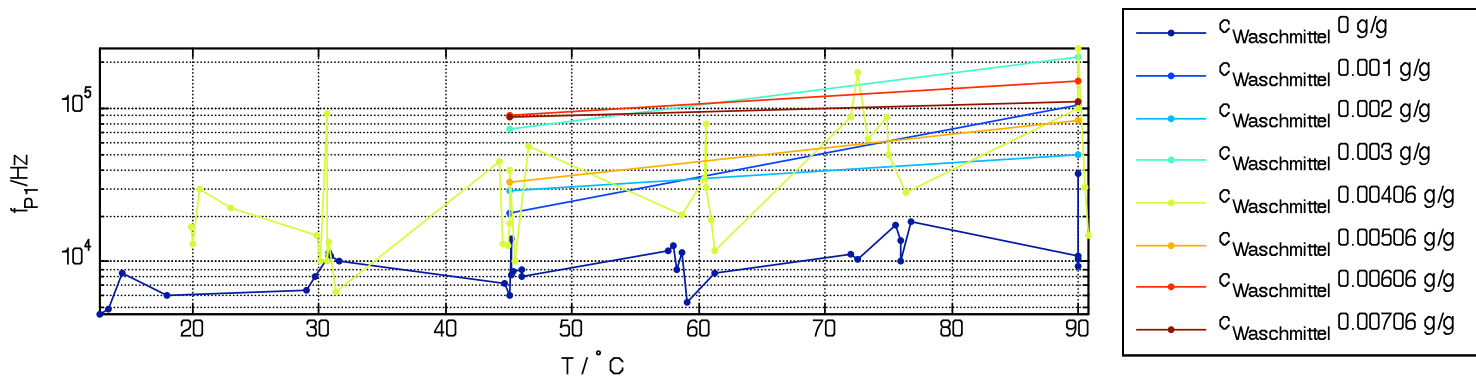

\section{Abbildung 14: Abhängigkeit der Frequenz des charakteristischen Punkt 1 von der Waschmittelkonzentration}

Die bisherigen Betrachtungen basieren im Wesentlichen auf der Auswertung der Leitfähigkeit. Typische Leitfähigkeitsmessgeräte bestimmen diese durch eine Impedanzmessung bei einer festen Frequenz. Durch die Nutzung der Impedanzspektroskopie kann im Gegensatz zu einer Messung bei einer festen Frequenz detektiert werden, ob die Bestimmung der Leitfähigkeit bei der richtigen Frequenz erfolgt. Die Frequenz kann bei jeder Messung individuell bestimmt werden. Somit kann die Leitfähigkeit für jede Messung exakt bestimmen. Abbildung 14 zeigt, wie die Frequenz des charakteristischen Punkt 1 in Abhängigkeit von der Waschmittelkonzentration um etwa eine Dekade variiert. Bei Bestimmung der Leitfähigkeit bei einer festen Frequenz im Frequenzbereich $1 \mathrm{kHz}$ bis $100 \mathrm{kHz}$ entsteht gegenüber der Bestimmung aus dem Realteil des charakteristischen Punkt 1 eine zusätzliche Leitfähigkeitsabweichung bis etwa $5 \%$.

\subsection{Einfluss von Verschmutzung}

Der Einfluss der Verschmutzung wird zunächst am Beispiel von Milch bei einer Temperatur von $40^{\circ} \mathrm{C}$ untersucht. Dazu wird für verschiedene Konzentrationen von Milch und Waschmittel ein Kennfeld aufgenommen. Anschließend wird Milch auf ein Gewebe gegeben und getrocknet. Das getrocknete Gewebe wird gewaschen und die Impedanz der Waschlauge gemessen. Ziel dabei ist, das Verhalten der Impedanz in Abhängigkeit der Konzentrationen zu bestimmen und festzustellen, ob die Messwerte für gewaschenes Gewebe mit denen einer Wasser-Waschmittel-Schmutz Gemisches übereinstimmen. Die abgebildeten Konzentrationen beschreiben dabei die das Verhältnis von Feststoffmasse bezogen auf die Masse des verwendeten Wassers. 


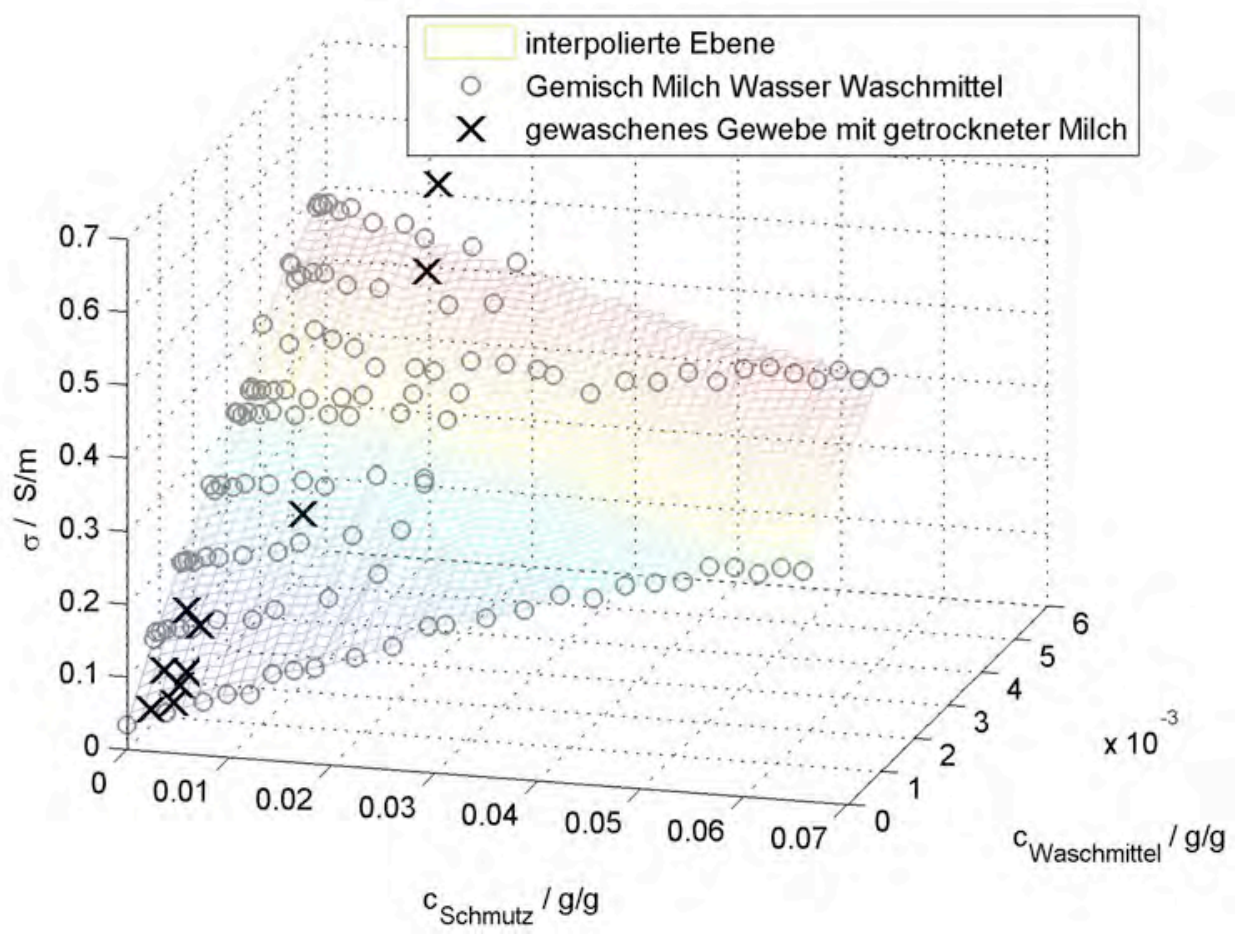

Abbildung 15: Verhalten der Leitfähigkeit in Abhängigkeit von Waschmittel und Schmutzkonzentration

Das Verhalten der Leitfähigkeit in Abbildung 15 zeigt dabei eine systematische Abhängigkeit. Erhöhte Waschmittel- und Schmutzkonzentration führen zu einer erhöhten Leitfähigkeit. Die Punkte für Messungen mit Testgewebe zeigen dabei eine Abweichung von etwa 20\% vom Messwert für die Mischung aus Wasser, Milch und Waschmittel. Das zeigt, dass eine Bestimmung der Verschmutzung von Textilien prinzipiell möglich ist. Bei bekanntem Zusammenhang zwischen Leitfähigkeit, Wasserhärte und Waschmittelkonzentration, die in den vorangegangenen Abschnitten gezeigt wurde, kann zusätzlich auf die Konzentration der Verschmutzung geschlossen werden. Dies ist insbesondere dann möglich, wenn die Verschmutzung wasserlöslich ist und die gebildeten lonen zur Leitfähigkeit beitragen.

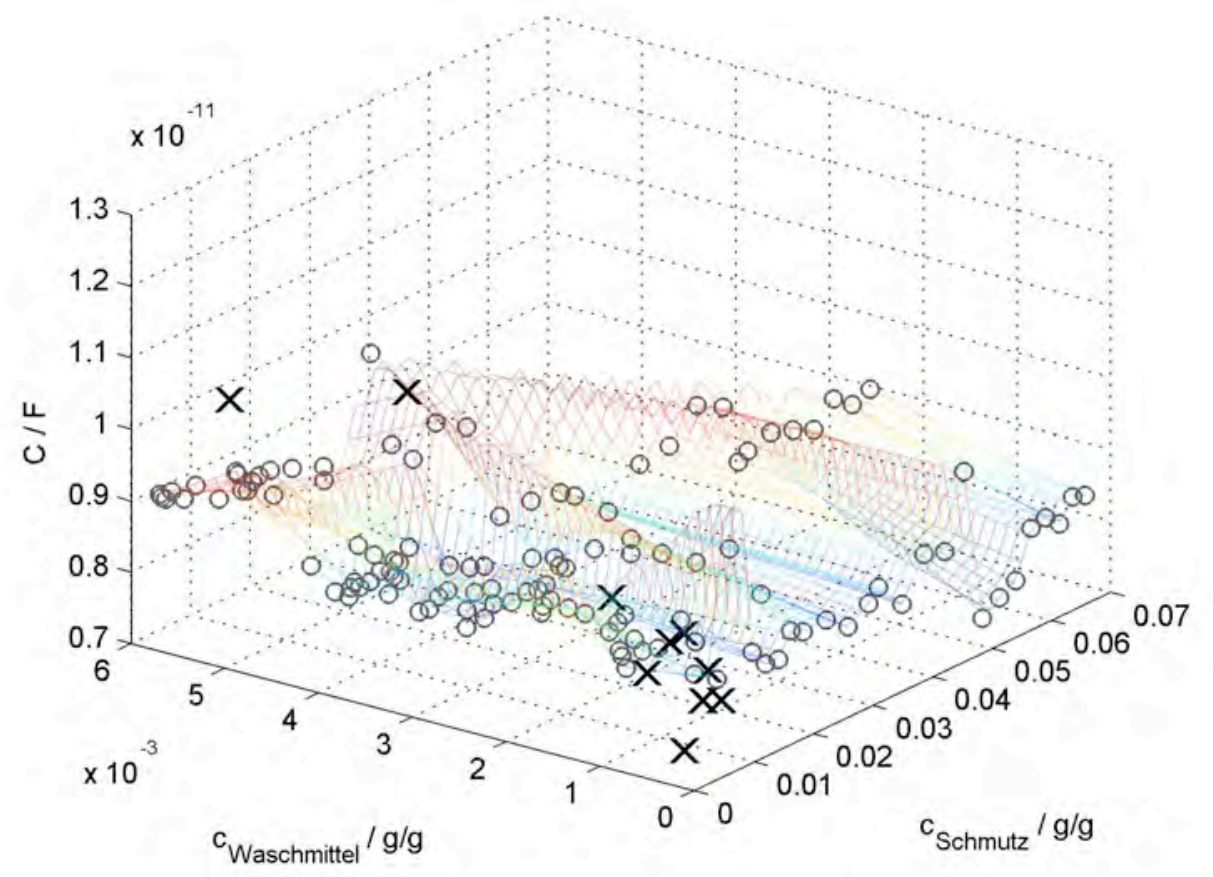

Abbildung 16: Verhalten der Kapazität in Abhängigkeit von Waschmittel und Schmutzkonzentration 


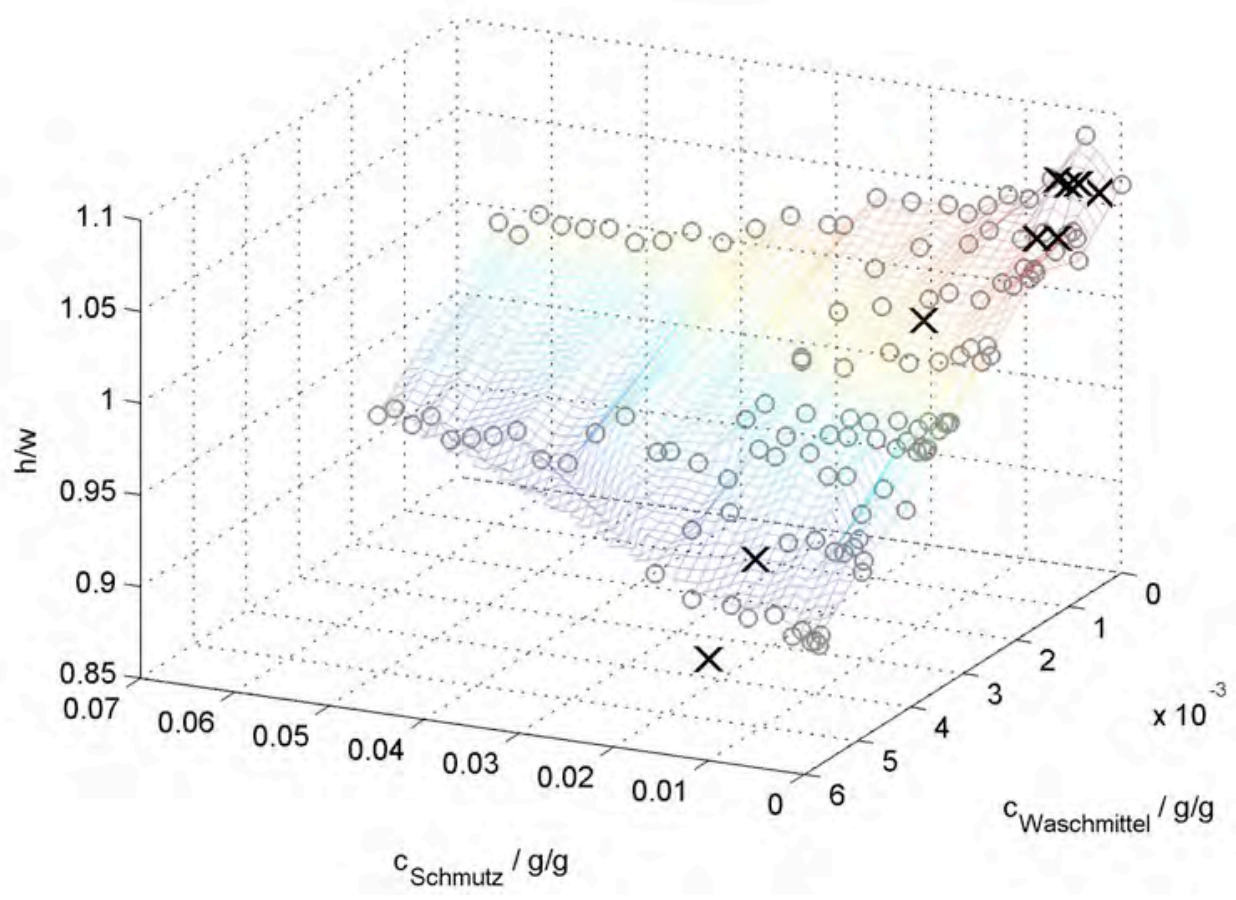

\section{Abbildung 17: Verhalten des Verhältnis von Höhe zu Weite in Abhängigkeit von Waschmittel- und Schmutzkonzentration}

Abbildung 16 zeigt die Abhängigkeit der Kapazität von der Waschmittel und Schmutzkonzentration. Dabei ist kein systematischer Zusammenhang zu erkennen. Die Messwerte streuen etwa 10\% um einen Wert von 10pF. Die Messabweichung des Messgerätes beträgt dabei etwa $1 \%$.

Abbildung 17 zeigt das Verhältnis von Höhe zu Weite. In Abhängigkeit von Waschmittel- und Schmutzkonzentration flacht der Halbkreis des Impedanzspektrums sehr stark ab, was zu einem abnehmenden Verhältnis von Höhe zu Weite führt. Dieses Verhalten ist vergleichbar zu den Ergebnissen in den Abschnitten 4.2.1 und 4.2.2. Höhere Waschmittel- und Schmutzkonzentration führen zu einem inhomogeneren Gemisch und damit zu einer Verteilung der Zeitkonstanten. Der beobachtete Halbkreis wird dabei zunehmend abgeflacht. Zur Messung der Waschmittel- und Schmutzkonzentration kann damit zusätzlich zur Leitfähigkeit das Verhältnis von Höhe zu Weite herangezogen werden. Dazu ist für dieses Verhältnis ein empirisches Modell aufzustellen und anhand von Messdaten in Abhängigkeit von der Temperatur zu parametrieren. Je nach erforderlicher Genauigkeit kann es sich dabei um ein lineares Modell oder auch ein nichtlineares Modell handeln. Durch die Invertierung des Modells können dann die Waschmittelkonzentration und die Schmutzkonzentration durch die Lösung eines Gleichungssystems bestimmt werden. Bei Nutzung eines linearen Modells kann für die Lösung des Gleichungssystems ein lineares Optimierungsverfahren genutzt werden.

\section{Zusammenfassung}

Die Ergebnisse zeigen, dass die Wasserhärtemessung und die Messung der Waschmittelkonzentration für Konzentrationen und Temperaturen, die für den Bereich Bekleidungsreinigung relevant sind, mit den vorgestellten Messprinzipien prinzipiell möglich sind. Ebenso ist eine Detektion von Verschmutzung prinzipiell möglich.

Die Messung der Wasserhärte konnte auf der Grundlage der Leitfähigkeit mit einer relativen Abweichung von etwa $20 \%$ bestimmt werden. Die Waschmittelkonzentration konnte mit einer relativen Abweichung von etwa $10 \%$ bezogen auf die empfohlene Konzentration bestimmt werden. Durch die Nutzung der Impedanzspektroskopie zur Bestimmung der Leitfähigkeit kann dabei die Genauigkeit gegenüber einer Festfrequenzmessung verbessert werden, da der Messwert anhand eines physikalisch sinnvoll gewählten charakteristischen Punktes des Impedanzspektrums bestimmt wird. Das Verhältnis von Höhe zu Weite des im Impedanzspektrum beobachteten Halbkreises bietet erweiterte Möglichkeiten zur Bestimmung der Waschmittelkonzentration und der Verschmutzung. Durch die Inhomogenität der Flüssigkeit entsteht eine Verteilung der Zeitkonstanten was zum Abflachen des Halbkreises führt. Damit kann eine deutliche Trennung zwischen den Effekten durch die Wasserhärte und die Temperatur und den Effekten durch Waschmittel und Verschmutzung erfolgen. Aus der Kapazität des Sensors konnte bisher keine sinnvoll 
nutzbare Information abgeleitet werden. Um die Abhängigkeit der Kapazität zu analysieren ist eine Optimierung des Messaufbaus erforderlich.

Zur Realisierung eines praktisch einsetzbaren Waschlaugensensors sind weiterhin Untersuchungen zum Verhalten verschiedener Waschmittel und der Langzeitstabilität erforderlich. Das erlaubt das Design eines robusten Sensors der resistent gegen Verschmutzung und unempfindlich gegen elektrische Störungen bei der Impedanzmessung ist. Ebenso ist eine dedizierte Messschaltung für die Impedanzspektroskopie erforderlich. In weiterführenden Arbeiten soll gezeigt werden, dass mit Hilfe geeigneter Vorrichtungen präzise und reproduzierbare Ergebnisse für eine Waschlaugenanalyse erreicht werden können.

\section{Literatur}

[1] Fröhling, S: Eine Waschmaschine die mitdenkt und mitspart. In: Sensor Magazin 3 (2011), S. 32-34

[2] Loch, C. ; Jung, C. ; Kersten, G.: Optical sensor for measuring opaqueness of washing or rinsing liquid. Patent, US 6,509,558, 2003

[3] Engel, Christian: Verfahren zur Temperaturkompensation der Meßwerte eines Trübungssensors in einer automatischen Waschoder Geschirrspülmaschine. Patent, DE 19521326 A 1, 1995

[4] Ivarsson, P. ; Johansson, M. ; Höjer, N.-E. ; Krantz-Rülcker, C. ; Winquist, F. ; Lundström, I.: Supervision of rinses in a washing machine by a voltammetric electronic tongue. In: Sensors and Actuators, B: Chemical 108 (2005), Nr. 1-2 SPEC. ISS., S. 851857. - DOI 10.1016/j.snb.2004.12.088

[5] Olsson, J. ; Ivarsson, P. ; Winquist, F.: Determination of detergents in washing machine wastewater with a voltammetric electronic tongue. In: Talanta 76 (2008), Nr. 1, S. 91-95. - DOI 10.1016/j.talanta.2008.02.028

[6] Chang, H.-C. ; Hwang, B.-J. ; Lin, Y.-Y. ; Chen, L.-J. ; Lin, S.-Y.: Measurement of critical micelle concentration of nonionic surfactant solutions using impedance spectroscopy technique. In: Review of Scientific Instruments 69 (1998), Nr. 6, S. 25142520. - DOI 10.1063/1.1148951

[7] Neto, J.M.G. ; Da Cunha, H.N. ; Neto, J.M.M. ; Ferreira, G.F.L.: Impedance spectroscopy analysis in a complex system: Sodium dodecyl sulfate solutions. In: Journal of Sol-Gel Science and Technology 38 (2006), Nr. 2, S. 191-195. - DOI 10.1007/s10971006-6350-1

[8] Nakamura, H. ; Sano, A. ; Matsuura, K.: Determination of critical micelle concentration of anionic surfactants by capillary electrophoresis using 2-naphthalenemethanol as a marker for micelle formation. In: Analytical Sciences 14 (1998), Nr. 2, S. 379-382. - DOI 10.2116/analsci.14.379

[9] Kurzweil, P. ; Fischle, H.-J.: A new monitoring method for electrochemical aggregates by impedance spectroscopy. In: Journal of Power Sources 127 (2004), Nr. 1-2, S. 331-340. - DOI 10.1016/j.jpowsour.2003.09.030

[10]Hamann, Carl H. ; Vielstich, Wolf: Elektrochemie. 3. Auflage. Weinheim : Wiley-VCH, 1997 